\title{
Generalization of Thermal and Mass Fluxes for the Flow of Differential Type Fluid with Caputo-Fabrizio Approach of Fractional Derivative
}

\author{
Asima Razzaque, ${ }^{1}$ Anam Rani, ${ }^{1}$ and Mudassar Nazar $\mathbb{D}^{2,3}$ \\ ${ }^{1}$ Department of Basic Science, Preparatory Year Deanship, King Faisal University Hofuf, Al Has, Hofuf, Saudia Arabia \\ ${ }^{2}$ School of Mathematical Sciences, University of Science and Technology of China, Hefei, Anhui, China \\ ${ }^{3}$ Centre for Advanced Studies in Pure and Applied Mathematics, Bahauddin Zakariya University, Multan, Pakistan
}

Correspondence should be addressed to Mudassar Nazar; mudassar_666@yahoo.com

Received 14 May 2021; Accepted 21 June 2021; Published 30 June 2021

Academic Editor: Dumitru Vieru

Copyright ( $) 2021$ Asima Razzaque et al. This is an open access article distributed under the Creative Commons Attribution License, which permits unrestricted use, distribution, and reproduction in any medium, provided the original work is properly cited.

\begin{abstract}
In this research work, generalized thermal and mass transports for the unsteady flow model of an incompressible differential type fluid are considered. The Caputo-Fabrizio fractional derivative is used for the respective generalization of Fourier's and Fick's laws. A MHD fluid flow is considered near a flat vertical surface subject to unsteady mechanical, thermal, and mass conditions at boundary. The governing equations of flow model are solved by integral transform, and closed form results for generalized momentum, thermal, and concentration fields are obtained. Generalized thermal and mass fluxes at boundary are quantified in terms of Nusselt and Sherwood numbers, respectively, and presented in tabular form. The significance of the physical parameters over the momentum, thermal, and concentration profiles is characterized by sketching the graphs.
\end{abstract}

\section{Introduction}

Fractional calculus has been expanding rapidly in the present time for the sake of its applications in the modeling and physical explanation of natural phenomenon. The noninteger derivatives of fractional order have been applied successfully to the generalization of fundamental laws of nature specially in the transport phenomenon.

Several approaches [1-4] of fractional derivatives have been proposed and utilized for the different proposes by many theorists from different fields of sciences and technology [5]. Imran et al. [6] considered two different approaches of fractional differential operators for the flow of MHD Newtonian fluid under the arbitrary boundary conditions, namely, Atangana and Caputo-Fabrizio. Kumar et al. [7] explained the Cauchy reaction diffusion equations by fractional calculus. Qureshi et al. [8] applied the fractional derivative to model a blood flow and discussed the concentration level of ethanol in blood circulation system.
Hristov [9] considered a steady-state heat conduction and obtained analytical solutions by applying the Caputo-Fabrizio approach of fractional derivative. Imran et al. $[10,11]$ considered the Caputo time fractional derivative to discuss the slippage flow over an exponentially accelerated plate and for the flow of differential fluid past stationary heated vertical plate. Ahmad et al. [12] compared two flow models, one with the power law kernel and the other with the nonsingular kernel. Khalid et al. [13] obtained the results for flow of micropolar fluid and applied the fractional derivative for heat and mass transport. Shukla et al. [14] presented a report regarding applications of fractional calculus. Kumar et al. [15] explored the results for free convectional motion with a uniform temperature through a porous media by utilizing the power, exponential, and Mittag-Leffler kernels of fractional operator. Singh et al. [16] constructed the dynamic fractional model to explain the smoking dynamics. Sun et al. [17] presented a collection of real world applications of fractional differential operators. Nazar et al. [18] 
discussed the double convectional flow via two approaches of noninteger operators and compared the obtained results of thermal, mass, and momentum profiles. Gomez et al. [19] solved fractional diffusion-advection equation and obtained the analytical solution for supper diffusion. Tran et al. [20] discussed the stabilities of fractional differential equation. Recently, Tuan et al. [21] endorsed the fractional calculus to demonstrate the transition model of COVID-19. Hristov et al. [22] applied the mixed time-space derivative to obtain the result for transient flow of non-Newtonian fluid. Saqib et al. [23], Haq et al. [24], and Imran et al. [25] utilized the fractional differential operator with the nonsingular kernel to obtain the fractional result for the flow of Jeffery, and second grade fluids. Some more investigations regarding Caputo-Fabrizio fractional derivatives are found in [26-28].

Hristov et al. [29] suggested the generalized transient thermal transport with damping contribution, by considering the Caputo-Fabrizio idea of nonsingular kernel. Aleem et al. [30], Sheikh et al. [31] and Ahmad et al. [32] followed the track suggested in [29] and applied it to describe the generalized heat and mass transfer flow. In light of the above motivational investigations, we are interested in discussing the generalized thermal and mass transports with heat generation and chemical reaction for the flow of second grade fluid through a porous media, with the existence of a magnetic field.

\section{Mathematical Formulation}

Suppose that the second grade fluid is lying in the vicinity of a vertical plate with ambient temperature $T_{\infty}$ and concentration level $C_{\infty}$. The orientation of the plate in the coordinate system is placed with $y$-axis becoming normal to the plane of plate as shown in Figure 1. Initially, the physical system containing boundaries and fluid is in complete equilibrium. Suddenly, plate starts moving with velocity $U_{0} f(t)$; at this moment, the temperature of plate and the concentration level near the plate rise or fall according to $T_{\infty}+\left(T_{w}-T_{\infty}\right) g(t)$ and $C_{\infty}+\left(T_{w}-C_{\infty}\right) h(t)$, respectively, where $f(\cdot), g(\cdot)$, and $h(\cdot)$ are arbitrary functions and satisfy $f(0)=0, g(0)=0$, and $h(0)=0$, respectively. The contribution of Lorentz force is also applied for the flow of fluid. Moreover, the induced magnetic field and heat dissipation are small and can be negligible. Subject to Boussinesq's approximation, the governing equations of respective flow take the following form $[29,30]$ :

$$
\begin{aligned}
& \text { Initial and boundary conditions: } \\
& \begin{array}{l}
u=0, T=T_{\infty}, C=C_{\infty}, \quad \text { at } t=0, \text { and, } y \in[0, \infty), \\
u=U_{0} f(t), \\
T=T_{\infty}+\left(T_{w}-T_{\infty}\right) g(t), \\
C=C_{\infty}+\left(T_{w}-C_{\infty}\right) h(t), \quad y=0, t>0, \\
u \longrightarrow 0, T \longrightarrow T_{\infty}, C \longrightarrow C_{\infty}, \quad \text { as } y \longrightarrow \infty, t>0 .
\end{array}
\end{aligned}
$$$$
\mu\left(1+\alpha_{1} \frac{\partial}{\partial t}\right) \frac{\partial^{2} u(y, t)}{\partial y^{2}}-\rho \frac{\partial u(y, t)}{\partial t}-\sigma B_{0}^{2} u(y, t)-\frac{\mu \phi}{K_{1}} u(y, t)=-\rho g \beta_{C}\left[C(y, t)-C_{\infty}\right]-\rho g \beta_{T}\left[T(y, t)-T_{\infty}\right]
$$$$
\rho C_{p} \frac{\partial T(y, t)}{\partial t}-Q\left[T(y, t)-T_{\infty}\right]=k \frac{\partial^{2} T(y, t)}{\partial y^{2}}
$$$$
\frac{\partial C(y, t)}{\partial t}-K_{r}\left[C(y, t)-C_{\infty}\right]=D \frac{\partial^{2} C(y, t)}{\partial y^{2}}
$$

\section{Generalized Model}

The thermal balance with heat generation is expressed as follows:

$$
\rho C_{p} \frac{\partial T(y, t)}{\partial t}=-\frac{\partial q}{\partial y}+Q\left[T(y, t)-T_{\infty}\right], \quad y, t>0,
$$

and the generalized thermal flux with damping effect $[29,30]$ is

$$
q(y, t)=-k_{1} \frac{\partial T(y, t)}{\partial y}-k_{2}{ }^{C F} D_{t}^{\alpha}(1-\alpha) \frac{\partial T(y, t)}{\partial y}, \quad y, t>0
$$

where ${ }^{C F} D_{t}^{\alpha}$ is the fractional differential operator suggested by Caputo-Fabrizio. The constant $h_{1}$ and $h_{2}$ are the effective heat conduction and elastic conduction parameters, respectively.

From equation (8), it is clear that, for $\alpha=1$, the classical thermal flux is recovered. Similarly, the molecular balance with chemical reaction is expressed as

$$
\frac{\partial C(y, t)}{\partial t}=-\frac{\partial J(y, t)}{\partial y}+K_{r}\left(C(y, t)-C_{\infty}\right), \quad y, t>0,
$$

and the generalized Fick's Law with damping effect $[29,30]$ is

$$
J(y, t)=-D_{1} \frac{\partial C(y, t)}{\partial y}-D_{2}^{C F} D_{t}^{\alpha}(1-\alpha) \frac{\partial C(y, t)}{\partial y}, \quad y, t>0,
$$




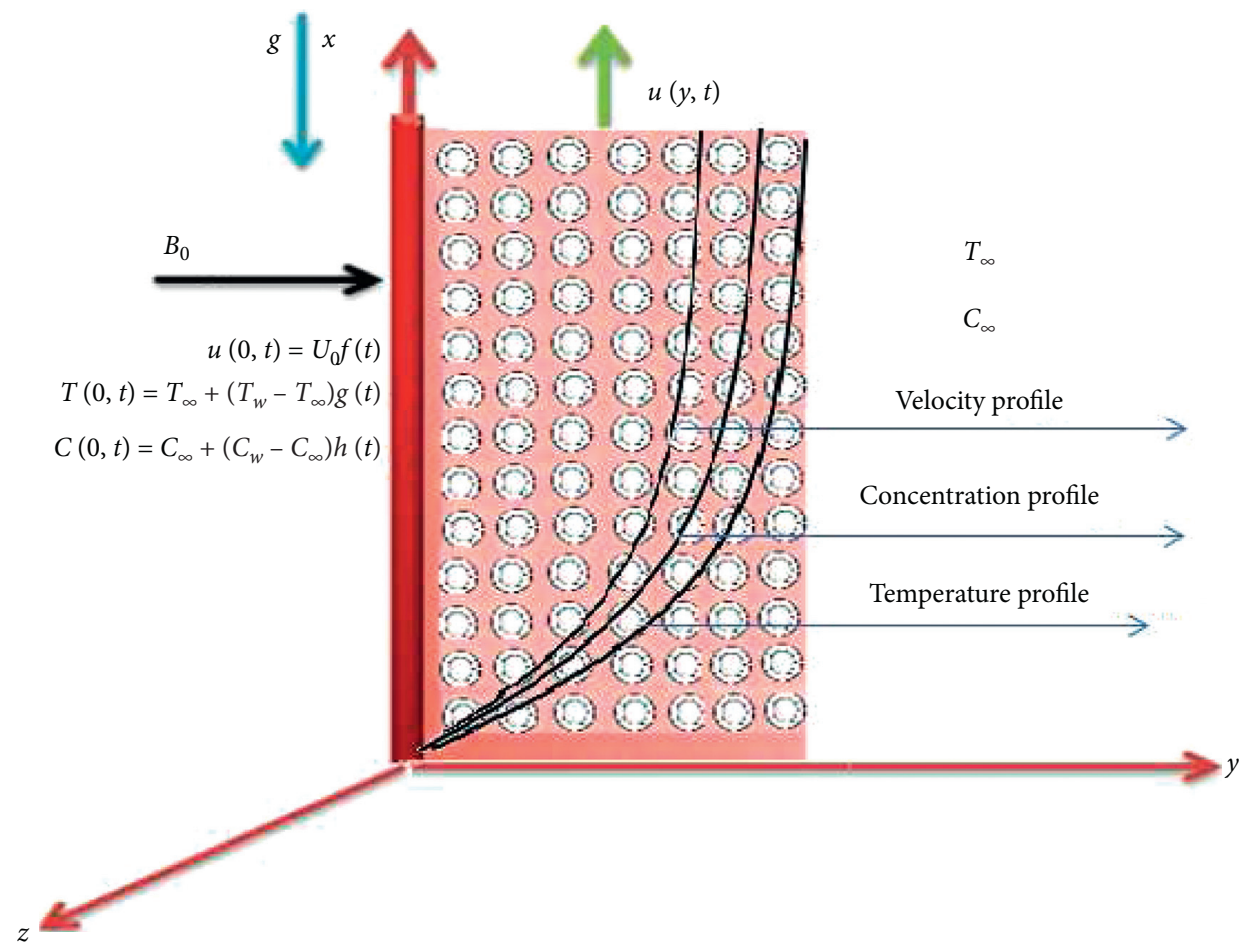

Figure 1: Flow geometry and coordinate system.

where $D_{1}$ and $D_{2}$ are the effective mass diffusion and the elastic diffusion, respectively.

Introduce the relations

$$
\begin{aligned}
v & =\frac{u}{U_{0}} \\
\eta & =\frac{y U_{0}}{\nu} \\
\tau & =\frac{t U_{0}^{2}}{v}, \\
\psi & =\frac{C-C_{\infty}}{C_{w}-C_{\infty}} \\
\varphi & =\frac{T-T_{\infty}}{T_{w}-T_{\infty}} \\
\left(1+\alpha_{2} \frac{\partial}{\partial \tau}\right) & \frac{\partial^{2} v(\eta, \tau)}{\partial \eta^{2}}-\frac{\partial v(\eta, \tau)}{\partial \tau}-M v(\eta, \tau)-\frac{1}{K} v(\eta, \tau)=-\operatorname{Gr} \varphi(\eta, \tau)-\operatorname{Gm} \psi(\eta, \tau)
\end{aligned}
$$

in equations (1), (4)-(6), and (7)-(10), to get the shape free model.

The momentum balance takes the following form:

Dimensionless thermal balance is

$$
\frac{\partial \varphi(\eta, \tau)}{\partial t}=\frac{1}{\operatorname{Pr}_{1}} \frac{\partial^{2} \varphi(\eta, \tau)}{\partial \eta^{2}}+\frac{1}{\operatorname{Pr}_{2}} C F D_{t}^{\alpha}(1-\alpha) \frac{\partial^{2} \varphi(\eta, \tau)}{\partial \eta^{2}}+Q_{0} \varphi(\eta, \tau) .
$$


Dimensionless diffusion equation is

$$
\frac{\partial \psi(\eta, \tau)}{\partial t}=\frac{1}{S c_{1}} \frac{\partial^{2} \psi(\eta, \tau)}{\partial \eta^{2}}+\frac{1}{S c_{2}}{ }^{C F} D_{t}^{\alpha}(1-\alpha) \frac{\partial^{2} \psi(\eta, \tau)}{\partial \eta^{2}}+K_{0} \psi(\eta, \tau)
$$

And the corresponding initial and boundary conditions are

$$
\begin{gathered}
v(\eta, 0)=0, \varphi(\eta, 0)=0, \psi(\eta, 0)=0, \quad \eta>0, \\
v(0, \tau)=f(\tau), \varphi(0, \tau)=g(\tau), \psi(0, \tau)=h(\tau), \quad \tau>0,
\end{gathered}
$$

$$
v(\eta, \tau) \longrightarrow 0, \varphi(\eta, \tau) \longrightarrow 0, \psi(\eta, \tau) \longrightarrow 0, \quad \text { as } \eta \longrightarrow 0
$$

where $\alpha_{2}=\left(\alpha_{1} U_{0}^{2} / \nu\right)$ is the dimensionless second grade parameter, $G m=\left(\left(g \beta_{C}\left(C_{w}-C_{\infty}\right)\right) / U_{0}^{3}\right)$ is the mass Grashof number, $G r=\left(\left(g \beta_{T}\left(T_{w}-T_{\infty}\right)\right) / U_{0}^{3}\right)$ is the thermal Grashof number, $M=\left(\nu \sigma /\left(\rho U_{0}^{2}\right)\right)$ is the magnetic parameter, $(1 / K)=\left(\nu^{2} \phi /\left(K_{1} U_{0}^{2}\right)\right)$ is the porosity parameter, $\operatorname{Pr}_{1}=$ $\left(\mu C_{p} / h_{1}\right)$ and $\operatorname{Pr}_{2}=\left(\mu C_{p} / h_{2}\right)$ are Prandtl numbers, $S c_{1}=$ $\left(\nu / D_{1}\right)$ and $S c_{2}=\left(\nu / D_{2}\right)$ are Schmidt numbers, $Q_{0}=\left(Q v /\left(\rho C_{p} U_{0}^{2}\right)\right)$ is the heat generation parameter, and $K_{0}=\left(K_{r} v / U_{0}^{2}\right)$ is the chemical reaction parameter.

\section{Solution of the Problem}

The solution of generalized model is obtained by endorsing the Laplace transform.

4.1. Generalized Temperature Field. Endorsing the Laplace transform to equation (16), an ordinary differential equation is obtained as

$$
q \bar{\varphi}(\eta, q)=\frac{1}{\operatorname{Pr}_{1}} \frac{\partial^{2} \bar{\varphi}(\eta, s)}{\partial \eta^{2}}+\frac{1}{\operatorname{Pr}_{2}}(1-\alpha)\left[\frac{q}{q(1-\alpha)+\alpha}\right] \frac{\partial^{2} \bar{\varphi}(\eta, q)}{\partial \eta^{2}}+Q_{0} \bar{\varphi}(\eta, q),
$$

with the following transformed boundary conditions:

$$
\bar{\varphi}(\eta, q)=G(q), \quad \text { and } \bar{\varphi}(\eta, q) \longrightarrow 0 \text {, as } \eta \longrightarrow \infty \text {. }
$$

Equation (18) is solved with conditions (19), and its solution is expressed as

$$
\bar{\varphi}(\eta, q)=G(q) \exp \left(-\eta \sqrt{\frac{\left(q-a_{0}\right)\left(q-Q_{0}\right)}{a_{1}\left(q+a_{2}\right)}}\right),
$$

where $a_{0}=(\alpha /(1-\alpha)), \quad a_{1}=\left(\left(\operatorname{Pr}_{1}+\operatorname{Pr}_{2}\right) / \operatorname{Pr}_{1} \operatorname{Pr}_{2}\right)$, and $a_{2}=\left(a_{0} \operatorname{Pr}_{2} /\left(\operatorname{Pr}_{1}+\operatorname{Pr}_{2}\right)\right)$.
Equation (20) is complicated, and it is not possible to invert the temperature field in $\mathrm{t}$-domain by ordinary formula of Laplace inverse. Therefore, the inversion algorithms, namely, Stehfest and Tzou, are utilized to invert the transformed temperature profile, and the obtained results are presented in Figure 2(a) for $g(t)=1$.

4.2. Generalized Concentration Field. Again endorsing the Laplace transform to equation (14), an ordinary differential equation is obtained as

$$
\begin{aligned}
& q \bar{\psi}(\eta, q)=\frac{1}{\mathrm{Sc}_{1}} \frac{\partial^{2} \bar{\psi}(\eta, s)}{\partial \eta^{2}}+\frac{1}{\mathrm{Sc}_{2}}(1-\alpha)\left[\frac{q}{q(1-\alpha)+\alpha}\right] \frac{\partial^{2} \bar{\psi}(\eta, q)}{\partial \eta^{2}}+K_{0} \bar{\psi}(\eta, q), \\
& \bar{\psi}(\eta, q)=H(q), \quad \text { and } \bar{\psi}(\eta, q) \longrightarrow 0, \text { as } \eta \longrightarrow \infty
\end{aligned}
$$

Equation (21) is solved with conditions (22), and its solution is expressed as

$$
\bar{\psi}(\eta, q)=H(q) \exp \left(-\eta \sqrt{\frac{\left(q-a_{0}\right)\left(q-K_{0}\right)}{b_{1}\left(q+b_{2}\right)}}\right),
$$

where

$$
b_{1}=\left(\left(S c_{1}+S c_{2}\right) / S c_{1} S c_{2}\right)
$$

and $b_{2}=\left(b_{0} S c_{2} /\left(S c_{1}+S c_{2}\right)\right)$

Equation (23) is complicated, and it is not possible to invert the temperature field in $\mathrm{t}$-domain by ordinary formula of Laplace inverse. Therefore, the inversion algorithms are 


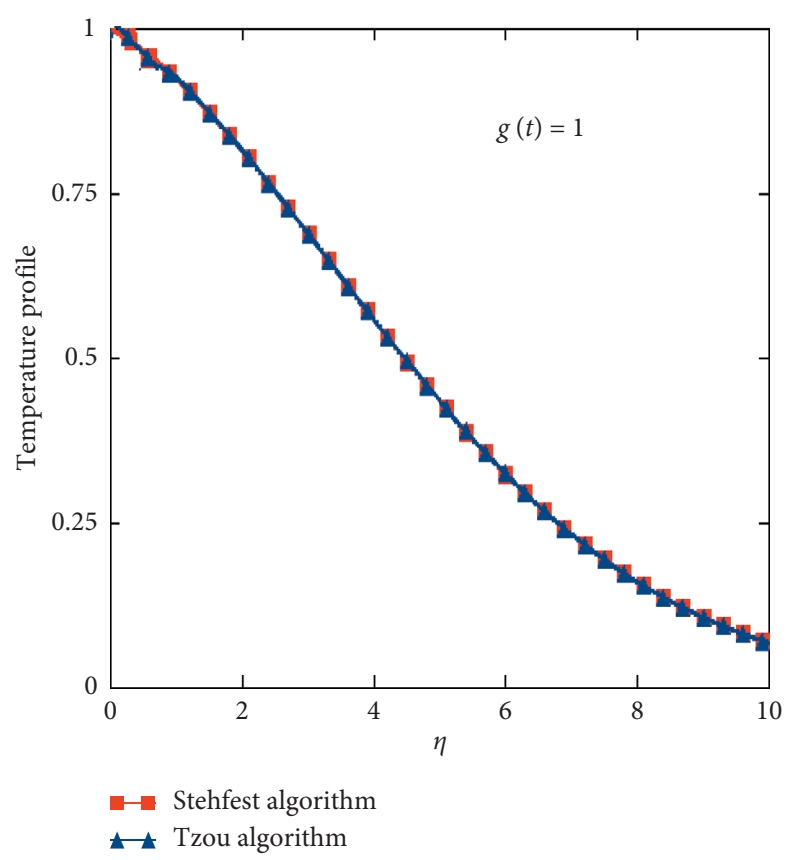

(a)

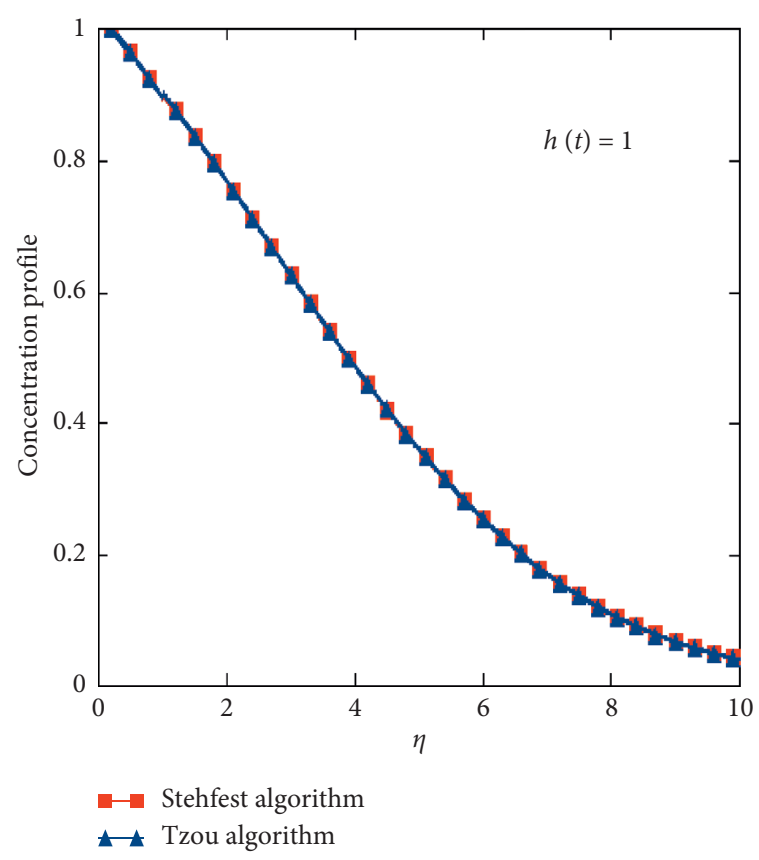

(b)

FIgURE 2: Profiles of inverted temperature and concentration with Stehfest's and Tzou's algorithms for $(\mathrm{a}) g(t)=1$ and $(\mathrm{b}) h(t)=1$.

utilized to obtain the temperature profile for $h(t)=1$, and the obtained results are presented in Figure 2(b).
4.3. Velocity Field with Generalized Thermal and Mass Transport. Equation (13) is converted to an ordinary differential equation via Laplace transform as

$$
\left(1+\alpha_{2} q\right) \frac{\partial^{2} \bar{v}(\eta, q)}{\partial \eta^{2}}-q \bar{v}(\eta, q)-M \bar{v}(\eta, q)-\frac{1}{K} \bar{v}(\eta, q)=-\operatorname{Gr} \bar{\varphi}(\eta, q)-\operatorname{Gm} \bar{\psi}(\eta, q)
$$

with corresponding transformed boundary conditions

$$
\bar{v}(0, q)=F(q), \quad \text { and } \bar{v}(\eta, q) \longrightarrow 0 \text { as } \eta \longrightarrow \infty .
$$

Equation (24), subject to condition (25), is solved for velocity field in $q$-domain as follows:

$$
\begin{aligned}
\bar{v}(\eta, q)= & F(q) \exp \left(-y \sqrt{\frac{q+M_{0}}{q+\alpha_{3}}}\right) \\
& +\frac{G r \alpha_{3} G(q)\left[\exp \left(-y \sqrt{\left(q+M_{0}\right) /\left(q+\alpha_{3}\right)}\right)-\exp \left(-y \sqrt{\left(\left(q+a_{0}\right)\left(q-Q_{0}\right)\right) / a_{1}\left(q+a_{2}\right)}\right)\right]}{\left(q+\alpha_{3}\right)\left(q-a_{0}\right)\left(q-Q_{0}\right)-a_{1}\left(q+a_{2}\right)\left(q+M_{0}\right)} \\
& +\frac{G m \alpha_{3} H(q)\left[\exp \left(-y \sqrt{\left(q+M_{0}\right) /\left(q+\alpha_{3}\right)}\right)-\exp \left(-y \sqrt{\left(\left(q+b_{0}\right)\left(q-K_{0}\right)\right) / b_{1}\left(q+b_{2}\right)}\right)\right]}{\left(q+\alpha_{3}\right)\left(q-b_{0}\right)\left(q-K_{0}\right)-b_{1}\left(q+b_{2}\right)\left(q+M_{0}\right)},
\end{aligned}
$$




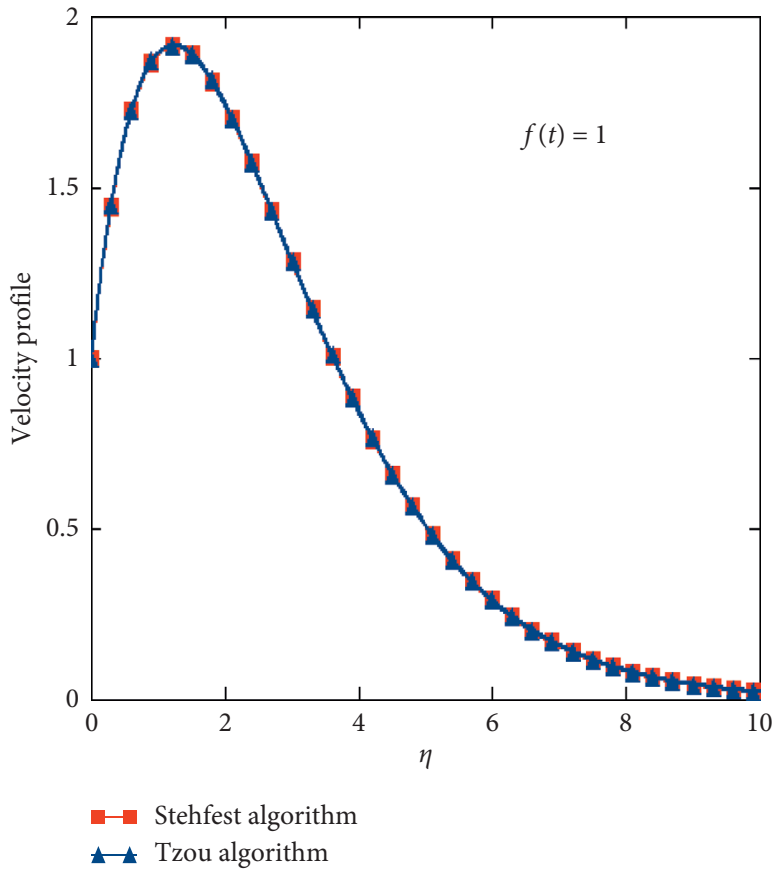

(a)

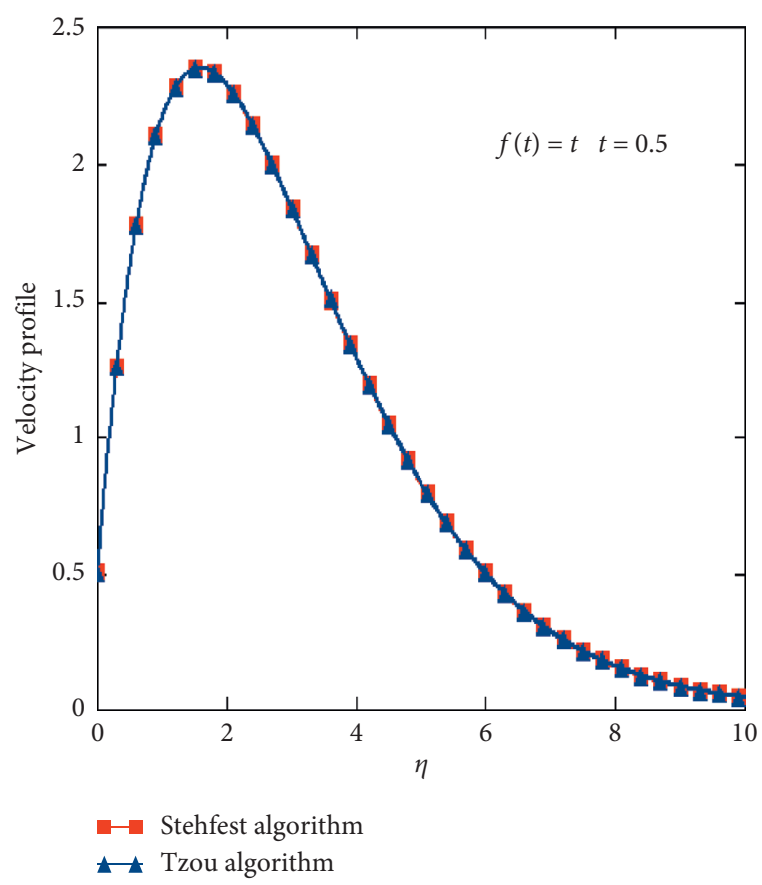

(b)

FIgure 3: Profile of inverted velocity with Stehfest's and Tzou's algorithms for (a) $f(t)=1$ and (b) $f(t)=t$.

where $\alpha_{3}=\left(1 / \alpha_{2}\right)$ and $M_{0}=M+(1 / K)$. Equation (26) is also complex, and it is inverted in t-domain with the help of inversion algorithms. The inverse Laplace of velocity for $f(t)=1$, and $f(t)=t$ is demonstrated in Figures 3(a) and $3(b)$.

\section{Results and Parametric Discussion}

This article is designed for the analysis of generalized thermal and mass transport flow of differential type fluid under generalized boundary conditions. The effects of magnetic field, heat source, and chemical reaction are also considered for flow model. The respective governing equations of flow model are solved analytically via integral transform method, and closed form expressions for field variables are attained.

The effects of potent parameters are also discussed graphically by plotting some graphs of for variation of appeared parameters. Figures 4(a) and 4(b) are sketched to explain the effect of fractional parameter $\alpha$ and $\operatorname{Pr}$ over the temperature profile, and it is noted that the temperature of fluid is raised with the developing values of $\alpha$. Also, it is detected that the temperature falls down with the increasing values of $\mathrm{Pr}$, because the fluid is thick, and momentum diffusivity is dominant to the thermal diffusivity for the greater Pr; therefore, fluid velocity slows down for increasing Pr. Figure 4(c) is drawn for three specification of $g(t)$, and it is noted that temperature profiles satisfy the boundary conditions.

In Figure 5, the subjectivity of concentration is explained for variations of $\alpha, \mathrm{Sc}$, and three specification for the $g(t)$. The same behavior of concentration is seen for respective parameters as seen in temperature profiles.

Velocity profile is outlined in Figure 6(a) due variation of fractional parameters $\alpha$. The figure pattern shows that the fluid gains more and more momentum as $\alpha$ tends to increase. The influence of Pr and Sc is signified in Figures 6(b) and 6(c). As Pr and Sc are quantified by the ratios of momentum diffusivity to thermal diffusivity and molecular diffusion coefficients, respectively, and for enhancing values of $\mathrm{Pr}$ and Sc referred to as the dominant momentum diffusivity, the fluid slows down for developing values of $\operatorname{Pr}$ and Sc.

Figure 7(a) is drawn to see the significance role of $\mathrm{Gr}$ for supporting the flow, and it is noted that the speed of fluid is elevated for developing values of Gr. As Gr is quantified by relative buoyancy force induced by the variations in temperature differences to the retarding force generates by the virtue of viscosity of the fluid, hence, for larger values of $\mathrm{Gr}$, there is more convectional current, so fluid speeds up. The effects of thermal generation parameter $Q_{0}$, and chemical reaction parameters $K_{0}$ are discussed in Figures $7(\mathrm{~b})$ and $7(c)$, and it is concluded that velocity profiles rise for enhancing values of $Q_{0}$ and $K_{0}$. 


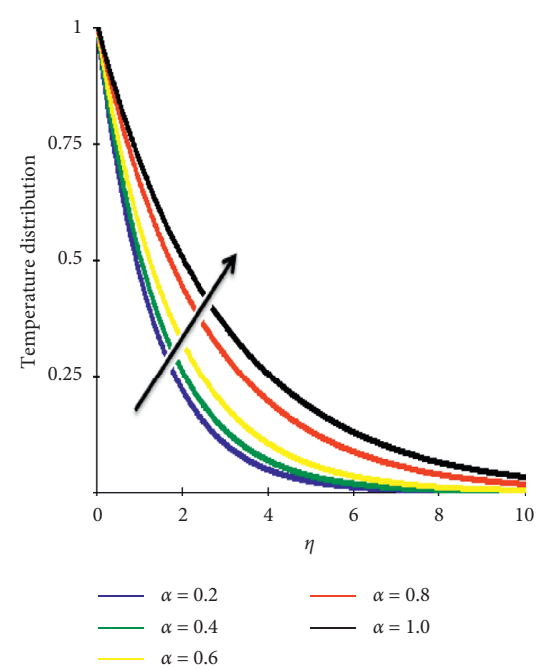

(a)
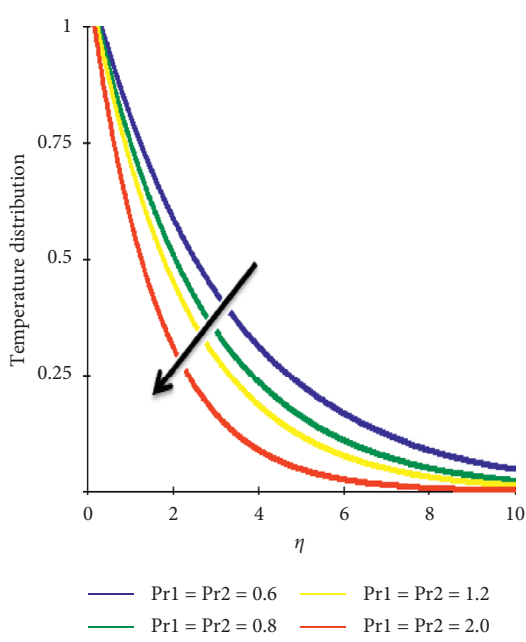

(b)

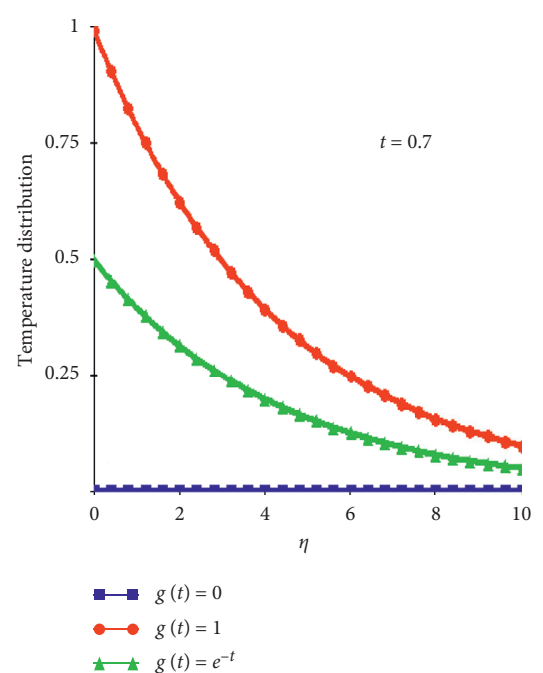

(c)

Figure 4: Sketch thermal profile for changing $\alpha$, $\operatorname{Pr}$, and $g(t)$.

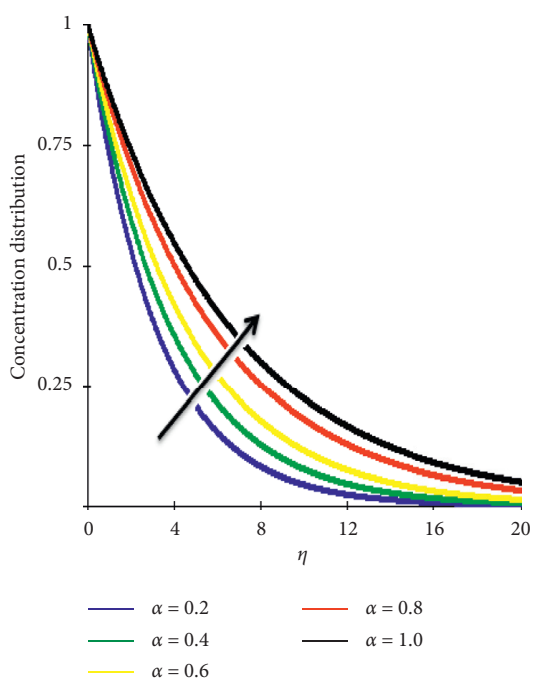

(a)
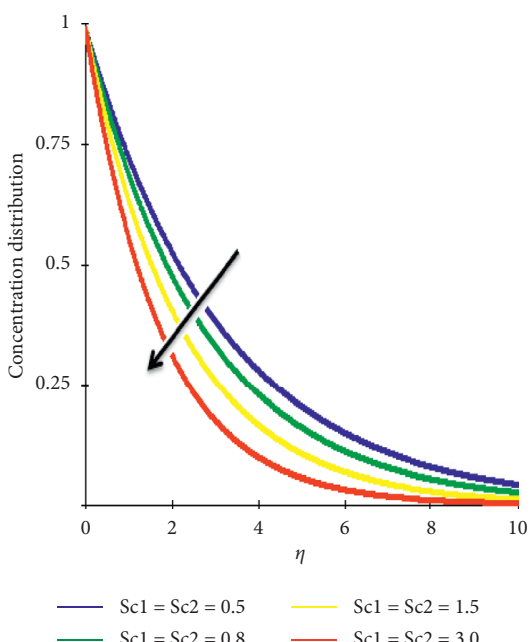

(b)

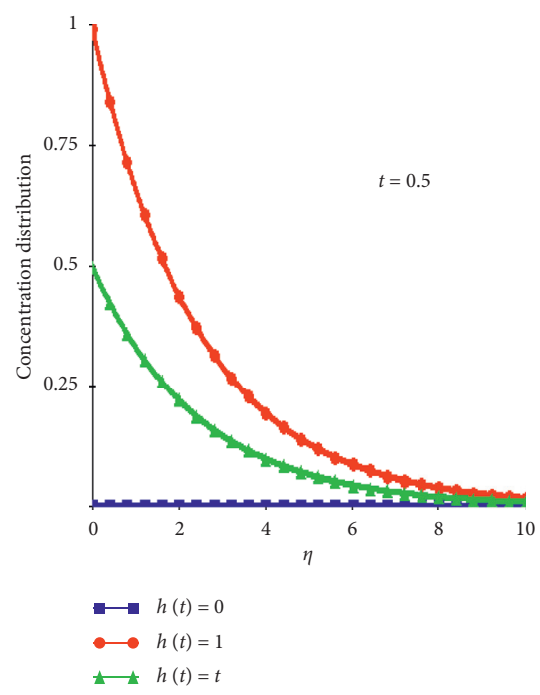

(c)

Figure 5: Sketch concentration profile for changing $\alpha$, Sc, and $h(t)$.

The subjectivity of magnetic parameter $M$ is highlighted in Figure 8(a) and from this figure, it is seen that profile lowers down for increasing values of $M$, because strong magnetic field creates more hindrance to the flow of fluid. Figure 8(b) shows the effect of $K$ and it is seen that fluid speeds up with the increasing values of $K$. The increasing values of $K$ refer to the decreasing effect of porosity, and hence, fluid velocity increases with the enhancing values of $K$. Figure $8(\mathrm{c})$ is plotted for three specifications of $f(t)$; from the profiles, it is clear that velocity profiles satisfy the boundary conditions for different $f(t)$. The present results for velocity and temperature are also compared with the existing results obtained by Sheh et al. [27] in Figures 9(a) and 9(b). The overlapping profiles confirm the validity of our results.

Further, heat and mass transfer at plate is discussed numerically in terms of Nusselt and Sherwood numbers, and results are presented in Tables 1 and 2. From these tables, it is clear that both Nusselt and Sherwood numbers are grown with elevating fractional parameter $\alpha$ for small time, while there is an opposite behavior for large time. 

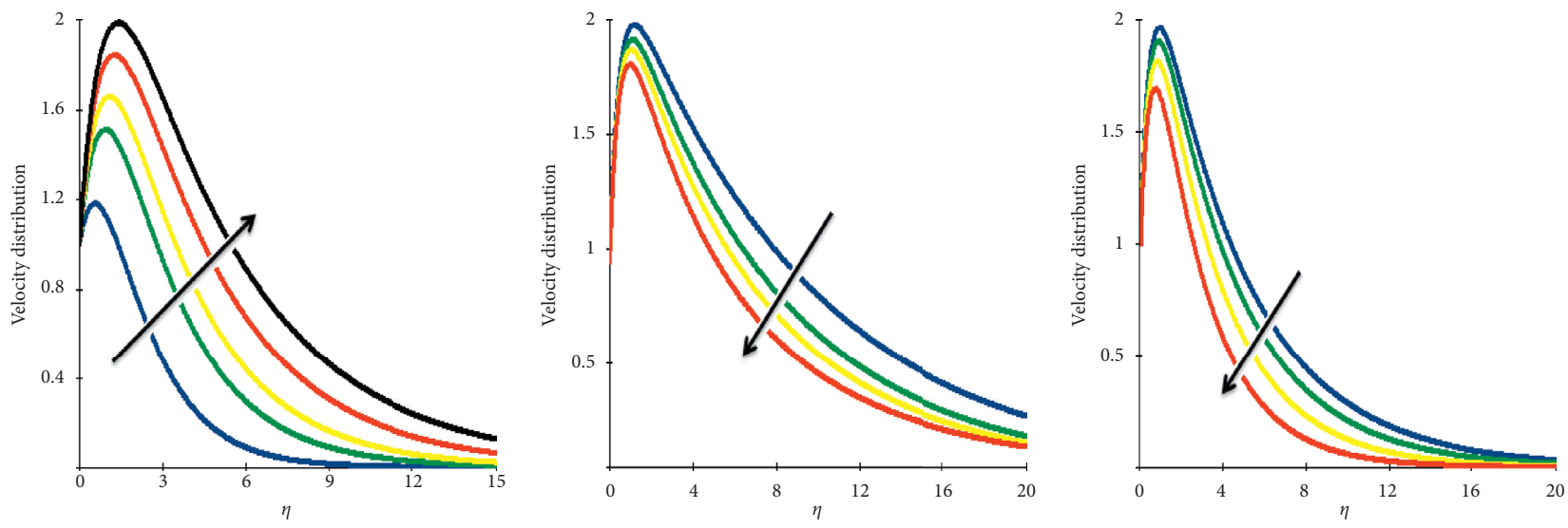

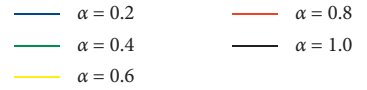

(a)

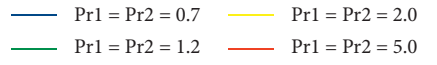

(b)

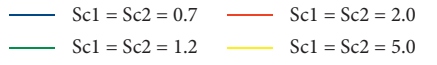

(c)

FIGURE 6: Sketch velocity profile for changing $\alpha, \mathrm{Pr}$, and Sc.

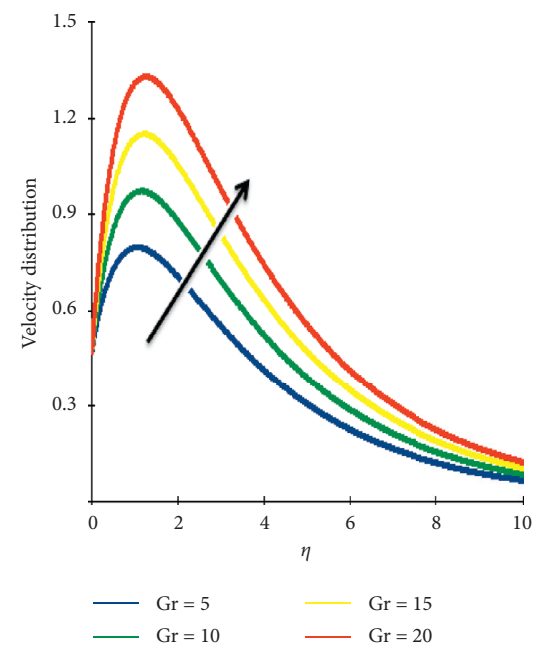

(a)

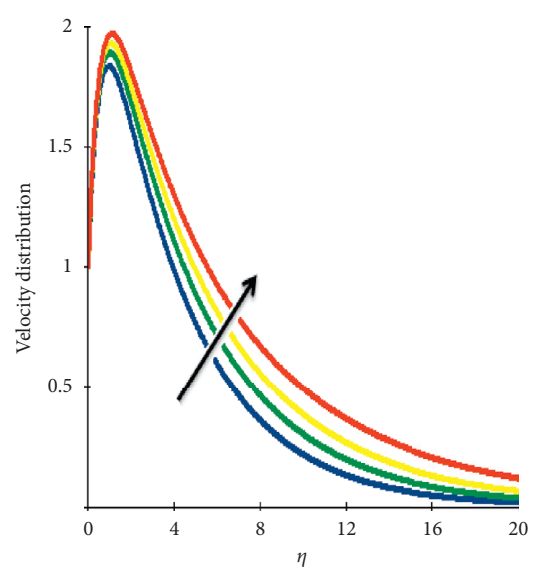

$\mathrm{Q} 0$
$\mathbf{\mathrm { Q }}=0.2$

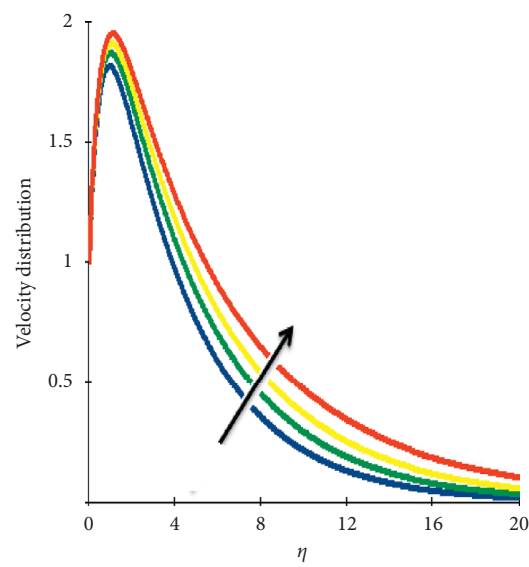

— $\mathrm{K} 0=0.2$

(b)

(c)

FIGURE 7: Sketch velocity profile for changing $G r, Q_{0}$, and $K_{0}$.

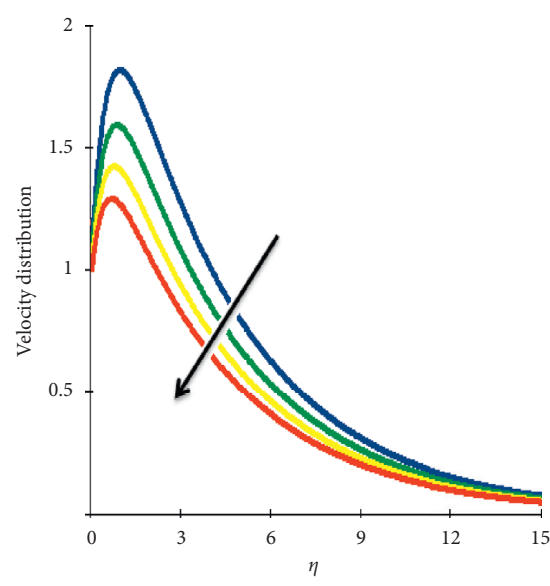

$\mathrm{M}=2$
$\mathrm{M}=3$

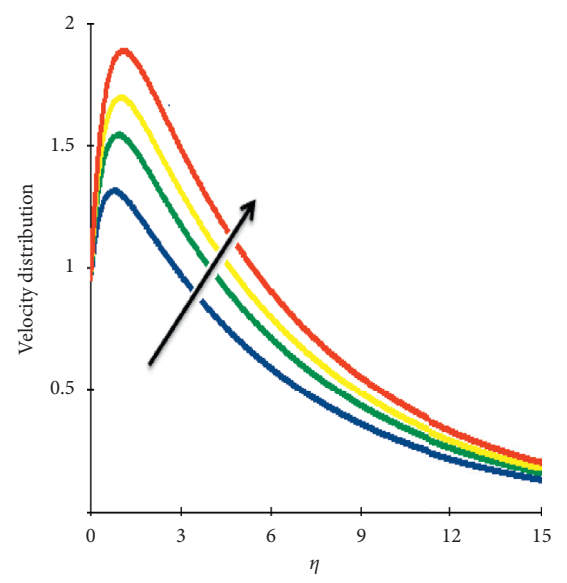

$-\mathrm{K}=0.2$

$\mathrm{K}=0.4$

$\mathrm{K}=0.6$

(b)

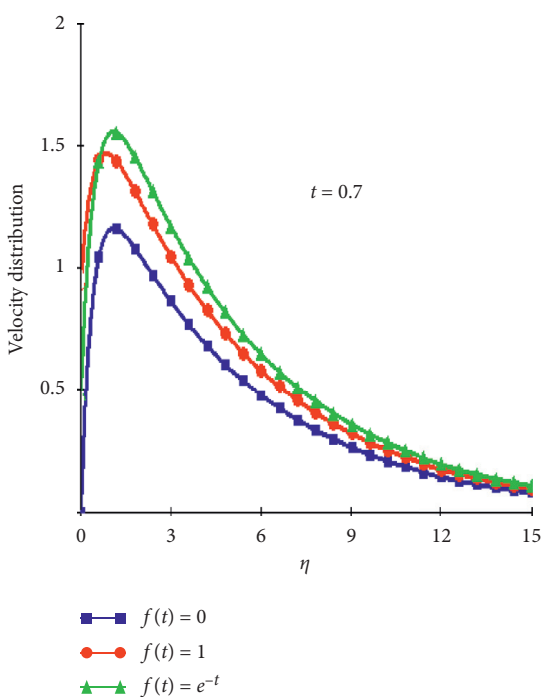

(c)

FIGURE 8: Sketch of velocity profile for changing values of $M, K$, and for $f(t)$. 


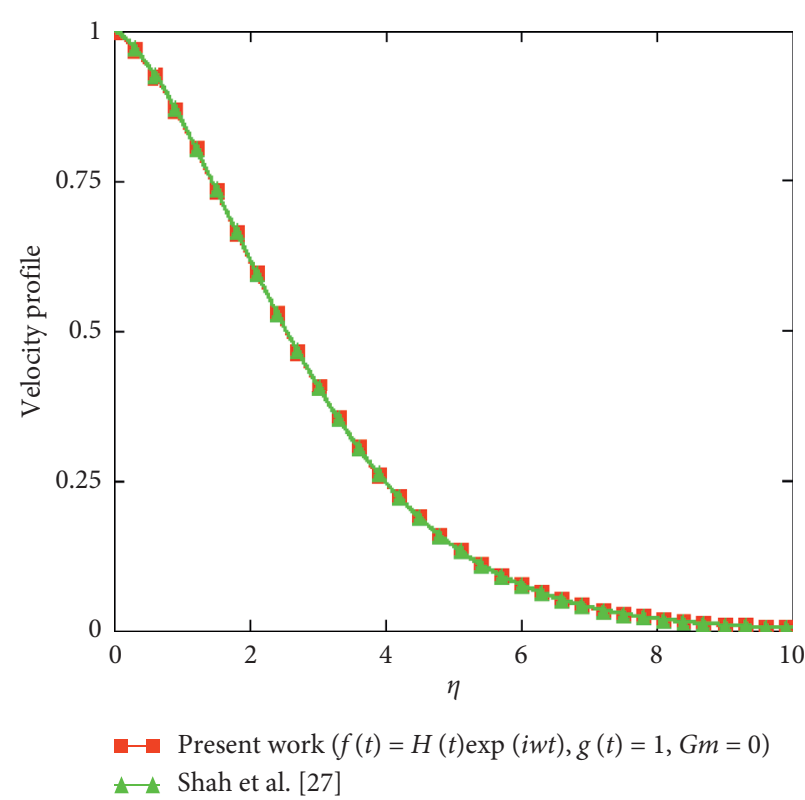

(a)

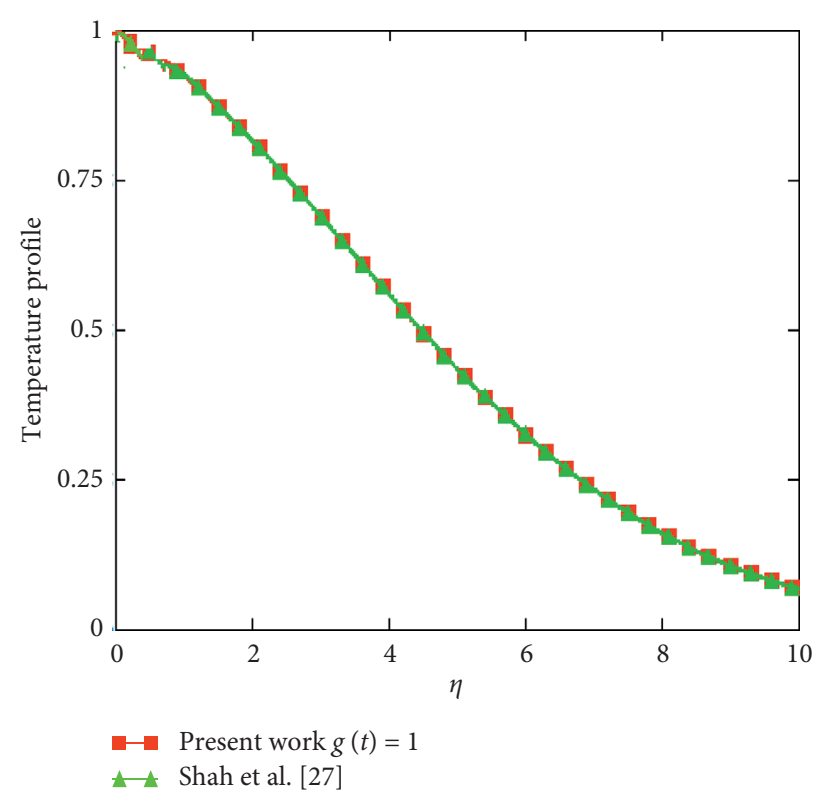

(b)

FIgURE 9: Sketch of velocity profile [ $f(t)=H(t) \exp (i w t), g(t)=1, G m=0]$ a comparison with Shah et al. [27].

TABLE 1: Subjectivity of Nusselt number due to $\alpha$ variation.

\begin{tabular}{lcccccc}
\hline$\alpha$ & $\operatorname{Pr}=2.0, t=0.05$ & $\operatorname{Pr}=2.0, t=0.5$ & $\operatorname{Pr}=2.0, t=0.3$ & $Q_{0}=0.3, t=0.05$ & $Q_{0}=0.3, t=0.5$ & $Q_{0}=0.3, t=3$ \\
\hline 0.1 & 1.28534684 & 1.63362404 & 1.58760351 & 1.366943741 & 0.367330281 & 0.10095136 \\
0.2 & 2.01663381 & 1.76776695 & 1.08576315 & 1.895117488 & 0.458735913 \\
0.3 & 2.93972368 & 1.91324675 & 0.83412160 & 2.748208635 & 0.543071730 & 0.10663781 \\
0.4 & 4.47358865 & 2.34431009 & 0.48029944 & 3.249259012 & 0.680603872 \\
0.5 & 5.49971941 & 2.70030862 & 0.27597129 & 3.593811291 & 0.898429253 & 0.09371372 \\
0.6 & 6.28539361 & 3.23449479 & 0.10950250 & 3.849001709 & 1.022261776 \\
0.7 & 6.92269321 & 3.67387267 & 0.07443112 & 4.046919659 & 1.143305599 & 0.07035624 \\
0.8 & 7.45715981 & 4.05046294 & 0.04832345 & 4.205461089 & 1.246011475 & 0.0360910366066 \\
0.9 & 7.91544825 & 4.38137291 & 0.03367067 & 4.335584398 & 1.335201230 & 0.11544229 \\
\hline
\end{tabular}

Table 2: Subjectivity of Sherwood number due to $\alpha$ variation.

\begin{tabular}{lcccccc}
\hline$\alpha$ & $\mathrm{Sc}=1.5, t=0.05$ & $\mathrm{Sc}=1.5, t=0.5$ & $\mathrm{Sc}=1.5, t=3$ & $K_{0}=0.5, t=0.05$ & $K_{0}=0.5, t=0.5$ & $K_{0}=0.5, t=3$ \\
\hline 0.1 & 1.36694374 & 1.07412976 & 0.21908902 & 1.16694374 & 1.35433928 & 0.21095136 \\
0.2 & 1.89511748 & 1.17645846 & 0.30983867 & 1.22263165 & 1.42893593 & 0.20663781 \\
0.3 & 2.74820863 & 1.81147383 & 0.43817805 & 2.45807253 & 1.54357105 & 0.20371372 \\
0.4 & 3.59381129 & 2.18028761 & 0.48989795 & 2.90622561 & 1.90603035 & 0.15091079 \\
0.5 & 3.59381129 & 2.43499766 & 0.57965507 & 3.21440254 & 2.50425241 & 0.11935624 \\
0.6 & 3.84900179 & 2.62480001 & 0.61967734 & 3.44265186 & 2.64561232 & 0.10743416 \\
0.7 & 4.04691969 & 2.77288204 & 0.72663608 & 3.61967501 & 2.74330559 & 0.08609199 \\
0.8 & 4.20546109 & 2.89214374 & 0.81975606 & 3.76147875 & 2.94601147 & 0.01366066 \\
0.9 & 4.33558438 & 2.99049848 & 0.90332718 & 3.87786456 & 3.00220123 & 0.00544229 \\
\hline
\end{tabular}

\section{Conclusion}

This investigation is designed to discuss the generalized thermal and mass transports and flow modeling for MHD second grade fluid subject to arbitrary conditions with the effect of heat generation and chemical reaction through a porous medium. The mathematical model is solved by integral transform method, and closed form relations for temperature, concentrations, and velocity fields are obtained. The effects of parameters for thermal and mass flow are discussed graphically. Also, thermal and mass 
fluxes at boundary of flow domain are explained numerically for the due variation of $\alpha$, and obtained results are given in the tabular form.

Some concluded bullets of this study are as follows:

(i) Temperature of the fluid is raised with incremental variation of $\alpha$ and $Q_{0}$, while it falls for the incremental variation of both $\operatorname{Pr}_{1}$ and $\operatorname{Pr}_{2}$

(ii) The thermal boundary conditions are also satisfied by temperature for different specifications of $g(t)$

(iii) The concentration level of the fluid is raised with incremental variation of $\alpha$ and $K_{0}$, whereas the level falls for the incremental variation of both $\mathrm{Sc}_{1}$ and $\mathrm{Sc}_{2}$

(iv) The concentration boundary conditions are also satisfied by the concentration for different specification of $h(t)$

(v) Velocity profile shows a growing trend for incremental increase in the values of $\alpha, \mathrm{Gr}, \mathrm{Gm}, Q_{0}, K_{0}$, and $K$ where as it retards for developing $\operatorname{Pr}_{1} \operatorname{Pr}_{2}$, $\mathrm{Sc}_{1}, \mathrm{Sc}_{2}$, and $M$

(vi) The Nusselt number is boosted with the increasing values of $\alpha$ by taking large values of time, while it falls down with the increasing $\alpha$ for small time

(vii) The Sherwood number grows with the increasing values of $\alpha$, for the large values of time, while it falls down with the increasing $\alpha$ for small time

\section{Data Availability}

All data are included within the article.

\section{Conflicts of Interest}

The authors declare that they have no conflicts of interest.

\section{Acknowledgments}

This project was sponsored by the Deanship of Scientific Research under Nasher Proposal No. 206207, King Faisal University.

\section{References}

[1] M. Caputo and M. Fabrizio, "A new definition of fractional derivative without singular kernel," Progress in Fractional Differentiation and Applications, vol. 1, no. 2, pp. 1-13, 2015.

[2] M. Caputo and M. Fabrizio, "Applications of new time and spatial fractional derivatives with exponential kernels," Progress in Fractional Differentiation and Applications, vol. 2, no. 1, pp. 1-11, 2016.

[3] D. Baleanu, A. Fernandez, and A. Akgül, "On a fractional operator combining proportional and classical differintegrals," Mathematics, vol. 8, no. 3, pp. 360-372, 2020.

[4] C. Milici, G. Drgnescu, and J. T. Machado, Introduction to Fractional Differential Equations, Springer, Berlin Germany, VII edition, 2019.

[5] C. H. Yu, "Fractional derivatives of some fractional functions and their applications," Asian Journal of Applied Science and Technology, vol. 4, no. 1, pp. 147-158, 2020.
[6] M. A. Imran, M. Aleem, M. B. Riaz, R. Ali, and I. Khan, "A comprehensive report on convective flow of fractional (ABC) and (CF) MHD viscous fluid subject to generalized boundary conditions," Chaos, Solitons \& Fractals, vol. 118, pp. 274-289, 2019.

[7] S. Kumar, A. Kumar, S. Abbas, M. A. Qurashi, and D. Baleanu, "A modified analytical approach with existence and uniqueness for fractional Cauchy reaction diffusion equations," Advances in Difference Equations, vol. 1, pp. 1-18, 2020.

[8] S. Qureshi, A. Yusuf, A. A. Shaikh, M. Inc, and D. Baleanu, "Fractional modeling of blood ethanol concentration system with real data application," Chaos: An Interdisciplinary Journal of Nonlinear Science, vol. 29, no. 1, Article ID 013143, 2019.

[9] J. Hristov, "Steady-state heat conduction in a medium with spatial non-singular fading memory: derivation of caputofabrizio space-fractional derivative from Canttaneo concept with Jeffrey's kernel and analytical solutions," Thermal Science, vol. 21, no. 2, pp. 827-839, 2017.

[10] M. A. Imran, S. Sarwar, and M. Nazar, "Influence of slip over an exponentially moving vertical plate with Caputo-time fractional derivative," Journal of Thermal Analysis and Calorimetry, 2020.

[11] M. A. Imran, I. Khan, M. Ahmad, N. A. Shah, and M. Nazar, "Heat and mass transport of differential type fluid with noninteger order time-fractional Caputo derivatives," Journal of Molecular Liquids, vol. 229, pp. 67-75, 2017.

[12] M. Ahmad, M. A. Imran, M. Aleem, and I. Khan, "A comparative study and analysis of natural convection flow of MHD non-Newtonian fluid in the presence of heat source and first-order chemical reaction," Journal of Thermal Analysis and Calorimetry, vol. 137, no. 5, pp. 1783-1796, 2019.

[13] A. Khalid, I. Khan, and S. Shafie, "Free convection flow of micropolar fluids over an oscillating vertical plate," Malaysian Journal of Fundamental and Applied Sciences, vol. 13, no. 4, pp. 654-658, 2017.

[14] R. K. Shukla and P. Sapra, "Fractional calculus and its applications for scientific professionals: a literature review," International Journal of Modern Mathematical Sciences, vol. 17, no. 2, pp. 111-137, 2019.

[15] D. Kumar, J. Singh, K. Tanwar, and D. Baleanu, "A new fractional exothermic reactions model having constant heat source in porous media with power, exponential and MittagLeffler laws," International Journal of Heat and Mass Transfer, vol. 138, pp. 1222-1227, 2019.

[16] J. Singh, D. Kumar, M. A. Qurashi, and D. Baleanu, "A new fractional model for giving up smoking dynamics," Advances in Difference Equations, vol. 88, pp. 1-16, 2017.

[17] H. Sun, Y. Zhang, D. Baleanu, W. Chen, and Y. Chen, "A new collection of real world applications of fractional calculus in science and engineering," Communications in Nonlinear Science and Numerical Simulation, vol. 64, pp. 213-231, 2018.

[18] M. Nazar, M. Ahmad, M. A. Imran, and N. A. Shah, "Double convection of heat and mass transfer flow of MHD generalized second grade fluid over an exponentially accelerated infinite vertical plate with heat absorption," Journal of Mathematical Analysis, vol. 8, pp. 1-10, 2017.

[19] F. Gómez, E. Escalante, C. Calderón, L. Morales, M. González, and R. Laguna, "Analytical solutions for the fractional diffusion-advection equation describing super-diffusion," Open Physics, vol. 14, no. 1, pp. 668-675, 2016.

[20] M. D. Tran, V. Ho, and H. N. Van, "On the stability of fractional differential equations involving generalized Caputo 
fractional derivative," Mathematical Problems in Engineering, vol. 2020, pp. 1-14, 2020.

[21] N. H. Tuan, H. Mohammadi, and S. Rezapour, "A mathematical model for COVID-19 transmission by using the Caputo fractional derivative," Chaos, Solitons \& Fractals, vol. 140, Article ID 110107, 2020.

[22] J. Hristov, "A transient flow of a non-Newtonian fluid modelled by a mixed time-space derivative: an improved integral-balance approach," Nonlinear Systems and Complexity, pp. 153-174, 2019.

[23] M. Saqib, F. Ali, I. Khan, N. A. Sheikh, S. A. A. Jan, and fnm Samiulhaq, "Exact solutions for free convection flow of generalized Jeffrey fluid: a Caputo-Fabrizio fractional model," Alexandria Engineering Journal, vol. 57, no. 3, pp. 1849-1858, 2018.

[24] S. Ul Haq, S. Jan, S. Ullah Jan, S. Inayat Ali Shah, I. Khan, and J. Singh, "Heat and mass transfer of fractional second grade fluid with slippage and ramped wall temperature using Caputo-Fabrizio fractional derivative approach," AIMS Mathematics, vol. 5, no. 4, pp. 3056-3088, 2020.

[25] M. A. Imran, N. A. Shah, M. Aleem, and I. Khan, "Heat transfer analysis of fractional second-grade fluid subject to Newtonian heating with Caputo and Caputo-Fabrizio fractional derivatives: a comparison," The European Physical Journal Plus, vol. 132, pp. 340-358, 2017.

[26] M. A. Khan, Z. Hammouch, and D. Baleanu, "Modeling the dynamics of hepatitis E via the Caputo-Fabrizio derivative," Mathematical Modelling of Natural Phenomena, vol. 14, no. 3, pp. 311-324, 2019.

[27] N. A. Shah and I. Khan, "Heat transfer analysis in a second grade fluid over and oscillating vertical plate using fractional Caputo-Fabrizio derivatives," The European Physical Journal C, vol. 76, no. 7, pp. 362-374, 2016.

[28] N. A. Sheikh, F. Ali, M. Saqib et al., "Comparison and analysis of the Atangana-Baleanu and Caputo-Fabrizio fractional derivatives for generalized Casson fluid model with heat generation and chemical reaction," Results in Physics, vol. 7, pp. 789-800, 2017.

[29] J. Hristov, "Transient heat diffusion with a non-singular fading memory from the Cattaneo constitutive equation with Jeffrey's kernel to the Caputo-Fabrizio time fractional derivative," Thermal Sciences, vol. 20, pp. 557-562, 2016.

[30] M. Aleem, M. Imran Asjad, M. S. R. Chowdhury, and A. Hussanan, "Analysis of mathematical model of fractional viscous fluid through a vertical rectangular channel," Chinese Journal of Physics, vol. 61, pp. 336-350, 2019.

[31] N. A. Sheikh, D. L. C. Ching, I. Khan, D. Kumar, and K. S. Nisar, "A new model of fractional Casson fluid based on generalized Fick's and Fourier's laws together with heat and mass transfer," Alexandria Engineering Journal, vol. 59, no. 5, pp. 2865-2876, 2020.

[32] M. Ahmad, M. A. Imran, and M. Nazar, "Mathematical modeling of $\left(\mathrm{CuAl}_{2} \mathrm{O}_{3}\right)$ water based Maxwell hybrid nanofluids with Caputo-Fabrizio fractional derivative," Advances in Mechanical Engineering, vol. 12, no. 9, pp. 1-11, 2020. 